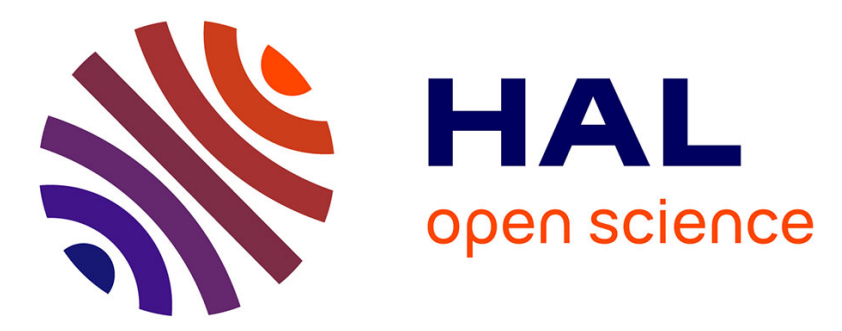

\title{
Bounding Deadline Misses in Weakly-Hard Real-Time Systems with Task Dependencies
}

Zain A. H. Hammadeh, Rolf Ernst, Sophie Quinton, Rafik Henia, Laurent Rioux

\section{- To cite this version:}

Zain A. H. Hammadeh, Rolf Ernst, Sophie Quinton, Rafik Henia, Laurent Rioux. Bounding Deadline Misses in Weakly-Hard Real-Time Systems with Task Dependencies. Design, Automation \& Test in Europe Conference \& Exhibition (DATE 2017), Mar 2017, Lausanne, Switzerland. hal-01426632

\section{HAL Id: hal-01426632 \\ https://hal.inria.fr/hal-01426632}

Submitted on 5 Jan 2017

HAL is a multi-disciplinary open access archive for the deposit and dissemination of scientific research documents, whether they are published or not. The documents may come from teaching and research institutions in France or abroad, or from public or private research centers.
L'archive ouverte pluridisciplinaire HAL, est destinée au dépôt et à la diffusion de documents scientifiques de niveau recherche, publiés ou non, émanant des établissements d'enseignement et de recherche français ou étrangers, des laboratoires publics ou privés.

\section{(c)(1)}

Distributed under a Creative Commons Attribution| 4.0 International License 


\section{Bounding Deadline Misses in Weakly-Hard Real-Time Systems with Task Dependencies}

\author{
Zain A. H. Hammadeh, Rolf Ernst \\ TU Braunschweig, Germany \\ \{hammadeh, ernst\}@ida.ing.tu-bs.de
}

\author{
Sophie Quinton \\ Inria Grenoble, France \\ sophie.quinton@inria.fr
}

\author{
Rafik Henia, Laurent Rioux \\ Thales Research \& Technology, France \\ \{rafik.henia, laurent.rioux\}@ thalesgroup.com
}

\begin{abstract}
Real-time systems with functional dependencies between tasks often require end-to-end (as opposed to task-level) guarantees. For many of these systems, it is even possible to accept the possibility of longer end-to-end delays if one can bound their frequency. Such systems are called weakly-hard.

In this paper we provide end-to-end deadline miss models for systems with task chains using Typical Worst-Case Analysis (TWCA). This bounds the number of potential deadline misses in a given sequence of activations of a task chain. To achieve this we exploit task chain properties which arise from the priority assignment of tasks in static-priority preemptive systems. This work is motivated by and validated on a realistic case study inspired by industrial practice and derived synthetic test cases.
\end{abstract}

\section{INTRODUCTION AND RELATED WORK}

Timing performance analysis of real-time systems with concurrently executing task chains is notoriously difficult due to the complexity of timing interference between tasks. This is all the more true when task chains are derived from communicating threads [9]. In this paper, we are interested in the analysis of end-to-end guarantees for weakly-hard systems with task dependencies, i.e., systems for which it is possible to accept the possibility of end-to-end deadline misses if one can bound their frequency [1].

We present a method to compute end-to-end deadline miss models for static-priority preemptive systems with task chains. This bounds the number of potential deadline misses in a given sequence of executions of a task chain. Our approach is an extension of Typical Worst-Case Analysis (TWCA) [8], [10], for which we exploit task chain properties derived from the priority assignment of tasks in a way similar to [9].

To the best of our knowledge, there is no state-of-the-art method for the computation of weakly-hard guarantees in realtime systems with task dependencies.

Extensive research has focused on the schedulability analysis of hard real-time systems with task dependencies. This includes approaches focusing on offset analysis [2] but also more general precedence models [3]. In [9], an upper bound on the end-to-end latency of task chains in real-time systems is presented, on which we will base our work in this paper.

In contrast, there is little in the literature regarding the analysis of weakly-hard systems. Initial attempts [4], [1] can only handle periodic tasks (or sporadic tasks but using a coarse

This work has been partially funded by the German Research Foundation (DFG) as part of the project "TypicalCPA" under the contract number TWCA ER168/30-1. interarrival time model) and no task dependencies. Recent work has focused on providing guarantees for systems with more complex activation patterns [8], [5], [10] and [6], mostly relying on the so-called TWCA approach. None of these, however, can handle task dependencies.

The paper is organized as follows: Section II introduces our system model and formulates the problem that we address. Then, Section III explains the basic principles of TWCA. Section IV proposes an improved version of the worst-case latency analysis of [9] which we use in $\mathrm{V}$ for the core contribution of our paper. Finally, Section VI shows our experimental results while Section VII proposes some conclusions.

\section{System Model}

We consider uniprocessor systems consisting of a finite set of $m$ disjoint task chains scheduled according to the Static Priority Preemptive (SPP) scheduling policy. A task chain is a sequence of distinct tasks which activate each other. Tasks in a system are required to belong to exactly one chain ${ }^{1}$. Formally, a task chain $\sigma_{a}, a \in[1, m]$, is defined by a finite sequence $\left(\tau_{a}^{1}, \tau_{a}^{2}, \ldots, \tau_{a}^{n}\right)$ of distinct tasks for some $n \in \mathbb{N}^{+}$, meaning that the output of $\tau_{a}^{i}$ is connected to the input of $\tau_{a}^{i+1}$ for $i \in[1, n-1]$. Every task chain $\sigma_{a}$ is assigned an activation model (see definition below) defining the frequency of arrival at the input of $\tau_{a}^{1}$; and a relative deadline $D_{a}$.

The tasks in $\sigma_{a}$ are denoted $\tau_{a}^{i}$, $\tau_{a}^{j}$ etc. Task $\tau_{a}^{i}$ denotes the $i$-th task in task chain $\sigma_{a}$. The number of tasks in $\sigma_{a}$ is denoted $n_{a}$. The first task in $\sigma_{a}$ is called the header task of $\sigma_{a}$ and the last one is called its tail task.

Figure 1 shows an example system with two task chains: $\sigma_{a}=\left(\tau_{a}^{1}, \tau_{a}^{2}, \tau_{a}^{3}, \tau_{a}^{4}, \tau_{a}^{5}, \tau_{a}^{6}\right), \sigma_{b}=\left(\tau_{b}^{1}, \tau_{b}^{2}, \tau_{b}^{3}\right)$.

We denote $\mathcal{C}$ the set of task chains. This set is partitioned into $\mathcal{S C}$ and $\mathcal{A C}$, which contain respectively the synchronous and asynchronous chains. Synchronous and asynchronous chains are specified in the same way but behave differently at execution: In a synchronous chain $\sigma_{a}$ an incoming activation cannot be processed until the previous instances of $\sigma_{a}$ have finished [9]. In an asynchronous chain $\sigma_{b}$ an incoming activation is processed independently from previous instances.

The activation models of task chains are defined using arrival curves as in e.g. [7], i.e., functions $\eta_{a}^{-}, \eta_{a}^{+}: \mathbb{N} \rightarrow \mathbb{N}$ such that for any time window $\Delta T, \eta_{a}^{+}(\Delta T)$ defines the

\footnotetext{
${ }^{1}$ To analyze systems that are not only made of disjoint task chains but also contain forks and joins (but no cycle), one can additionally define paths, i.e. sequences of distinct task chains. This is out of the scope of this paper.
} 


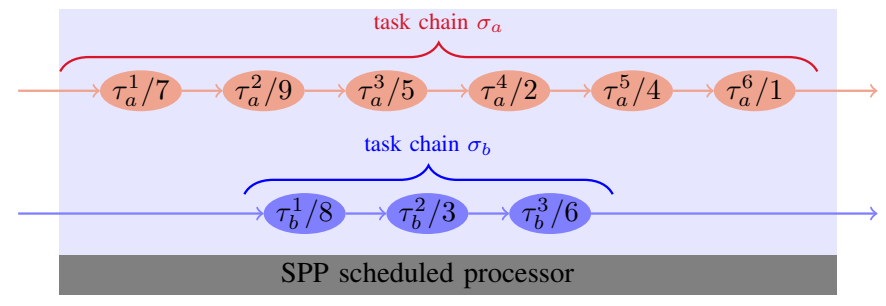

Figure 1. A system task structure with chains and task priorities

maximum number of activations of chain $\sigma_{a}$ that might occur within $\Delta T$, and $\eta_{a}^{-}(\Delta T)$ the minimum (in this paper we only use $\eta_{a}^{+}$). We will also need the pseudo-inverse representation of arrival curves, namely $\delta_{b}^{-}, \delta_{b}^{+}: \mathbb{N} \rightarrow \mathbb{N}$, such that $\delta_{b}^{-}(k)$ (respectively $\delta_{b}^{+}(k)$ ) defines the minimum (respectively maximum) time that might pass between the first and the last activation in any sequence of $k$ consecutive activations of $\sigma_{b}$.

A task $\tau_{a}^{i}$ is defined by: (1) an arbitrary priority $\pi_{a}^{i}$ and (2) an upper bound on its execution time $C_{a}^{i}$ (we take 0 as a lower bound). The notation $\pi_{a}^{i}>\pi_{b}^{j}$ is used to denote that $\tau_{a}^{i}$ has higher priority than $\tau_{b}^{j}$. As a result, $\tau_{a}^{i}$ may preempt $\tau_{b}^{j}$ when it arrives. We also use the notation $\pi_{a}^{i} \geqslant \pi_{b}^{j}$.

The timing behavior of a task $\tau_{a}^{i}$ is an infinite sequence of instances defined by: an arrival time, possible preemption delays and a finish time. Preemption delays are due to the task being blocked by higher priority tasks from other task chains, but also by higher priority tasks from the same chain if it is asynchronous. In contrast, tasks in a synchronous chain cannot be preempted by other tasks of the same chain, even if they have higher priority. Task $\tau_{a}^{i}$ finishes latest after having been scheduled for $C_{a}^{i}$ units of time.

The timing behavior of a task chain $\sigma_{a}$ is an infinite sequence of task chain instances, where a task chain instance is made of one instance of each task in the chain such that the finish time of task $\tau_{a}^{i}$ corresponds to the arrival time of task $\tau_{a}^{i+1}$ (assuming $\tau_{a}^{i}$ is not the last task in the chain). The arrival of task $\tau_{a}^{1}$ follows the activation model of $\sigma_{a}$.

The latency of an instance of a task chain $\sigma_{a}$ is the time interval between the activation of the header task of $\sigma_{a}$ and the finish time of the tail task of $\sigma_{a}$. The worst-case latency of $\sigma_{a}$ is the maximum latency over all instances of $\sigma_{a}$. An instance of $\sigma_{b}$ is said to miss its deadline if its latency exceeds the relative deadline of $\sigma_{b}$. This can happen in weakly-hard real-time systems. We consider a simple, deadline-agnostic scheduler that does not anticipate, monitor or react to deadline misses but instead runs every instance to completion, independent of whether a deadline miss has occurred or not.

As usual in TWCA, we suppose that deadline misses are caused by rarely activated sporadic chains, e.g., interrupt service routines or recovery chains. These chains cause transient overload, increasing chain latencies which may cause deadline misses, hence their name: overload chains. We assume that the set of overload chains is identified and denoted $\mathcal{C}_{\text {over }}$.

Definition 1. A deadline miss model for a task chain $\sigma_{b}$ is a function $d m m_{b}: \mathbb{N}^{+} \rightarrow \mathbb{N}$ such that $d m m_{b}(k)$ bounds the maximum number of deadline misses in a window of $k$ consecutive executions of $\sigma_{b}$.

In this paper, we address the problem of computing DMMs of task chains in systems which contain overload task chains.

\section{PRINCIPLE OF TYPICAL WORST-CASE ANALYSIS}

Typical Worst-Case Analysis (TWCA) is a technique to compute deadline miss models which bound the number of deadline misses in a sequence of activations of a given task. TWCA applies to systems of independent tasks which may occasionally miss deadlines due to overload tasks. We recall here the principle of TWCA and refer to [10] for more detail.

Formally, a Deadline Miss Model (DMM) for a task $\tau_{i}$ is a function $d m m_{i}: \mathbb{N}^{+} \rightarrow \mathbb{N}$ such that $d m m_{i}(k)$ bounds the maximum number of deadline misses that $\tau_{i}$ may experience out of a sequence of $k$ consecutive executions. The DMM computation is based on the analysis of unschedulable combinations, i.e., sets of overload tasks which, when activated together, may lead to a deadline miss. More formally, a combination, denoted $\bar{c}$, is a set of overload tasks. $\bar{c}$ is schedulable (with respect to $\tau_{i}$ ) if an instance of $\tau_{i}$ is guaranteed to meet its deadline as long as only tasks in $\bar{c}$ experience overload activations in its level- $i$ busy window, where a level-i busy window is a maximal time interval during which the processor has activations of $\tau_{i}$ or higher priority tasks pending.

Let us consider a sequence of $k$ activations of a given task $\tau_{i}$ and focus on the computation of $d m m_{i}(k)$. Note that the sequence may span multiple busy windows. The activation model of the overload tasks bounds the number of activations of these tasks (also called overload activations) which may arrive during the considered sequence. Assuming we have all unschedulable combinations at hand, the problem is then to find how to assign overload activations to busy windows so as to pack as many unschedulable combinations as possible into the level- $i$ busy windows under consideration. Therefore the problem becomes a multi-dimensional knapsack problem.

Example. Figure 2 illustrates two possible packings of overload activations into 5 busy windows. Every row corresponds to one overload task while every column corresponds to one busy window of $\tau_{i}$. The number of activations per line is constrained by the activation models of the overload tasks. The number of deadline misses associated to a given packing depends on how many columns are unschedulable combinations. Here, any combination containing more than one task is unschedulable.
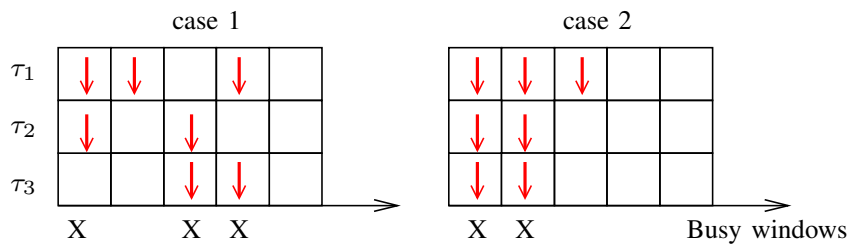

Figure 2. Packing combinations into busy windows ( $\mathrm{X}=$ deadline miss).

So far, TWCA can only handle independent tasks. In the rest of this paper we show how the state-of-the-art approach can be generalized to systems with task chains. 


\section{LATENCY ANALYSIS REVISITED}

Let us first revisit the worst-case latency analysis of systems with task chains [9]. Consider two chains $\sigma_{a}$ and $\sigma_{b}$. To quantify the interference of $\sigma_{a}$ on $\sigma_{b}$ we distinguish two cases:

1) some tasks in $\sigma_{a}$ have lower priority than all tasks in $\sigma_{b}$; in that case, $\sigma_{a}$ will be blocked by $\sigma_{b}$ every time it reaches one of those tasks.

2) In any other case, $\sigma_{a}$ is said to arbitrarily interfere with $\sigma_{b}$. This means that every time $\sigma_{a}$ is triggered, we suppose that it may entirely execute before $\sigma_{b}$ can be scheduled again. As we will see later, there is no guarantee however that this will happen.

Definition 2. A chain $\sigma_{a}$ is said to be deferred by chain $\sigma_{b}$ if

$$
\exists i \in\left[1, n_{a}\right], \pi_{a}^{i}<\min \left\{\pi_{b}^{j}\right\}_{j=1}^{n_{b}}
$$

Otherwise it is arbitrarily interfering with $\sigma_{b}$.

The set of chains deferred by $\sigma_{b}$ is denoted $\mathcal{D C}(b)$ and the set of chains arbitrarily interfering with $\sigma_{b}$ is denoted $\mathcal{I C}(b)$.

For a chain $\sigma_{a}$ which is arbitrarily interfering with $\sigma_{b}$, interference on $\sigma_{b}$ can be directly derived from the number of activations of $\sigma_{a}$. If $\sigma_{a}$ is, however, deferred by $\sigma_{b}$, then interference is defined based on the concept of segment of $\sigma_{a}$ w.r.t. $\sigma_{b}$. Intuitively, a segment of $\sigma_{a}$ w.r.t. $\sigma_{b}$ represents a subchain of $\sigma_{a}$ that may interfere with $\sigma_{b}$.

Definition 3. A segment of $\sigma_{a}$ w.r.t $\sigma_{b}$ is a maximal subchain $\left(\tau_{a}^{i}, \tau_{a}^{i+1}, \ldots, \tau_{a}^{i+k}\right)$ of $\sigma_{a}, i \in\left[1, n_{a}\right]$ and $k \in\left[0, n_{a}-1\right]$, with the convention ${ }^{2}$ that task identifiers should be read modulo $n_{a}$ and such that

$$
\forall l \in[0, k], \pi_{a}^{i+l} \geqslant \min \left\{\pi_{b}^{j}\right\}_{j=1}^{n_{b}}
$$

Note that we (conservatively) assume that a segment may span over two instances of $\sigma_{b}$. $S_{a}^{b}$ denotes all such segments.

Example. Chain $\sigma_{a}$ in Figure 1 has 2 segments w.r.t. chain $\sigma_{b}$ : $\left(\tau_{a}^{1}, \tau_{a}^{2}, \tau_{a}^{3}\right)$ and $\left(\tau_{a}^{5}\right)$. Note that $\tau_{a}^{4}$ and $\tau_{a}^{6}$ have lower priority than $\tau_{b}^{2}$ and are therefore not part of any segment.

Definition 4. The critical segment of a chain $\sigma_{a}$ deferred by $\sigma_{b}$, denoted $s_{a, b}^{c r i t}$, is the segment $\left(\tau_{a}^{i}, \tau_{a}^{i+1}, \ldots, \tau_{a}^{i+k}\right)$ of $\sigma_{a}$ w.r.t. $\sigma_{b}$ that maximizes computation time, i.e., $\sum_{0 \leqslant l \leqslant k} C_{a}^{i+l}$.

Definition 5. Consider an asynchronous chain $\sigma_{a}$. We denote:

- $s_{a}^{\text {header }}$ the subchain $\left(\tau_{a}^{1}, \tau_{a}^{2}, \ldots, \tau_{a}^{i}\right)$ where $i \in\left[1, n_{a}-1\right]$ is the smallest integer such that $\tau_{a}^{i+1}$ has the lowest priority in $\sigma_{a}$. If $\tau_{a}^{1}$ has the lowest priority then $s_{a}^{\text {header }}$ is empty.

- if $\sigma_{a}$ is deferred by $\sigma_{b}$ then we denote $s_{a, b}^{\text {header }}$ the header segment of $\sigma_{a}$ w.r.t. $\sigma_{b}$ defined as the subchain $\left(\tau_{a}^{1}, \tau_{a}^{2}, \ldots, \tau_{a}^{i}\right)$ where $i \in\left[1, n_{a}-1\right]$ is the smallest integer such that $\tau_{a}^{i+1}$ has lower priority than all tasks in $\sigma_{b}$.

We now revisit the worst-case latency analysis introduced in [9] and propose a description that is similar to worst-case response-time analysis as explained in [8].

\footnotetext{
${ }^{2}$ That is, if $i+l>n_{a}$ then it should be read $(i+l) \bmod n_{a}$.
}

Definition 6. $A \sigma_{b}$-busy-window is a maximal time interval during which (at least) one instance of $\sigma_{b}$ is pending, i.e., it has been activated but has not finished yet.

Definition 7. The $q$-event busy time of a chain $\sigma_{b}$ is the maximum time it may take to process $q$ activations of $\sigma_{b}$ within $a \sigma_{b}$-busy-window starting with the first of these q activations.

Theorem 1. The q-event busy time of $\sigma_{b}$ is bounded by

$$
\begin{aligned}
B_{b}(q)= & q \times C_{b} \\
& +\max \left(0, \eta_{b}^{+}\left(B_{b}(q)\right)-q\right) \times C_{s_{b}^{\text {header }}} \text { if } \sigma_{b} \in \mathcal{A C} \\
& +\sum_{\sigma_{a} \in \mathcal{I C}(b)} \eta_{a}^{+}\left(B_{b}(q)\right) \times C_{a} \\
& +\sum_{\sigma_{a} \in \mathcal{A} \mathcal{C} \cap \mathcal{D C}(b)} \eta_{a}^{+}\left(B_{b}(q)\right) \times C_{s_{a, b}^{\text {header }}}+\sum_{s \in S_{a}^{b}} C_{s} \\
& +\sum_{\sigma_{a} \in \mathcal{S C} \cap \mathcal{D C}(b)} C_{s_{a, b}^{\text {crit }}}
\end{aligned}
$$

where $C_{x}$ denotes the sum of the execution time bounds of the tasks in segment or chain $x$.

Proof. The above equation is made of five components:

1) The first line corresponds to the time needed to actually perform the $q$ computations;

2) The second component accounts for the interference of additional activations of $\sigma_{b}$ which may arrive while the $q$ activations under consideration are being processed. Note that these instances will at most interfere until they have to execute the lowest priority task in $\sigma_{b}$. This component only applies to asynchronous chains;

3) The third element represents the interference from arbitrarily interfering chains, synchronous or asynchronous;

4) The fourth line deals with interference from deferred, asynchronous chains. Instances can arbitrarily queue up which allows the header segment to interfere arbitrarily. For all other segments at most one instance can be backlogged because tasks between segments have lower priority than tasks within segments. Each such instance can interfere for at most one segment (see below).

5) The fifth component in the equation accounts for the interference from deferred, synchronous chains. Here only one instance per chain may interfere for at most one segment (see below).

The correctness of the last two components in Equation (1) relies on the following property.

Lemma 1. Tasks of a chain $\sigma_{a}$ that are in different segments cannot execute instances corresponding to the same chain instance in the same $\sigma_{b}$-busy-window.

Proof. Segments are maximal sequences of tasks with a priority higher than or equal to the lowest priority task, say $\tau_{b}^{i}$, in $\sigma_{b}$. This means that between two segments of $\sigma_{a}$ there is at least one task, say $\tau_{a}^{j}$, that has lower priority than $\tau_{b}^{i}$. In order to execute these two segments for the same instance of $\sigma_{a}$, one has to execute $\tau_{a}^{j}$. Since $\tau_{a}^{j}$ has lower priority than all 


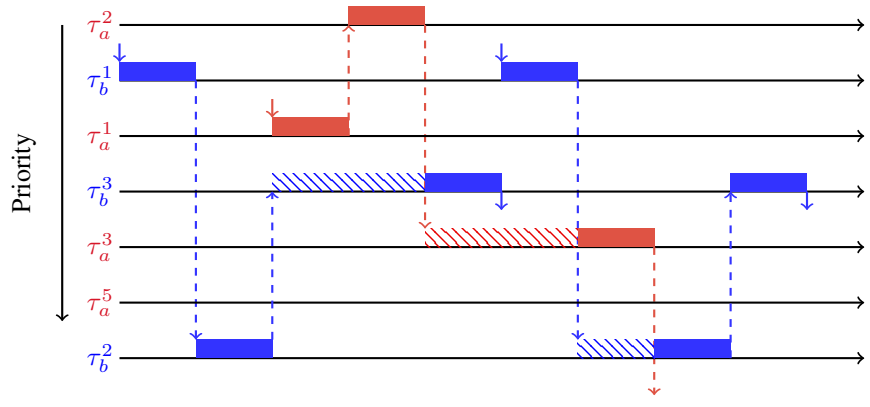

Figure 3. Number of impacted busy windows of chain $b$.

the tasks in $\sigma_{b}$, this can only happen after $\sigma_{b}$ closes its current $\sigma_{b}$-busy-window.

Theorem 2. The maximum number of activations of $\sigma_{b}$ in a $\sigma_{b}$-busy-window is

$$
K_{b}=\min \left\{q \geqslant 1 \mid B_{b}(q) \leqslant \delta_{b}^{-}(q+1)\right\}
$$

The latency of a task chain $\sigma_{b}$ is bounded by

$$
W C L_{b}=\max _{q \in\left[1, K_{b}\right]}\left\{B_{b}(q)-\delta_{b}^{-}(q)\right\}
$$

Proof. This proof proceeds exactly as the proofs in [8].

The main objective of TWCA is to bound the number of deadlines misses of a task chain $\sigma_{b}$ which may be caused by an activation at the input of an overload task chain $\sigma_{a}$. For that, we need to know over how many $\sigma_{b}$-busy-windows a instance of $\sigma_{a}$ may span.

We already know that, in a chain $\sigma_{a}$, the execution of tasks corresponding to the same instance of $\sigma_{a}$ cannot take place in the same $\sigma_{b}$-busy-window if those tasks are in different segments. This implies that an instance of $\sigma_{a}$ spans over at least as many $\sigma_{b}$-busy-windows as there are segments of $\sigma_{a}$ w.r.t. $\sigma_{b}$.

Note that there is no guarantee that a segment of $\sigma_{a}$ will be executed within one $\sigma_{b}$-busy-window. As an example, in Figure 3 the execution of segment $\left(\tau_{a}^{1}, \tau_{a}^{2}, \tau_{a}^{3}\right)$ spans over two $\sigma_{b}$-busy-windows. We therefore introduce the notion of active segment, which applies to subsegments which are guaranteed to be executed in the same $\sigma_{b}$-busy-window.

Definition 8. An active segment of $\sigma_{a}$ w.r.t $\sigma_{b}$ is a subchain ${ }^{3}$ of a segment $\left(\tau_{a}^{i}, \tau_{a}^{i+1}, \ldots, \tau_{a}^{i+k}\right)$ of $\sigma_{a}$ where $i \in\left[1, n_{a}\right]$ and $k \in\left[0, n_{a}-i\right]$ such that

$$
\forall l \in[1, k], \pi_{a}^{i+l} \geqslant \pi_{b}^{\text {tail }}
$$

where $\tau_{b}^{\text {tail }}$ denotes the tail task of $\sigma_{b}$.

Example. In Figure 1, chain $\sigma_{a}$ has three active segments: $\left(\tau_{a}^{1}, \tau_{a}^{2}\right),\left(\tau_{a}^{3}\right),\left(\tau_{a}^{5}\right)$.

Lemma 2. The execution of an active segment of $\sigma_{a}$ w.r.t. $\sigma_{b}$ cannot span over more than one $\sigma_{b}$-busy-window.

\footnotetext{
${ }^{3}$ Here, $i+l$ is always smaller than or equal to $n_{a}$.
}

Proof. Once the execution of an active segment of $\sigma_{a}$ w.r.t. $\sigma_{b}$ has started, $\tau_{b}^{\text {tail }}$ will not be able to execute because the active segment is blocking it or a task preceding it, and therefore the current $\sigma_{b}$-busy-window cannot be closed, until the whole segment has finished executing.

This lemma is illustrated in Figure 3, where every active segment of chain $\sigma_{a}$ executes within one $\sigma_{b}$-busy-window.

Note that an active segment is part of a segment in the sense of Definition 3. As a result, we easily conclude from Lemma 1 and 2 that two active segments of chain $\sigma_{a}$ may be executed within one $\sigma_{b}$-busy-window if and only if they are part of the same segment of $\sigma_{a}$.

\section{TWCA FOR TASK CHAINS}

We now have all the ingredients needed to show how we extend TWCA to handle task chains. We follow here the same approach as the one for systems with independent tasks explained in Section III. For the rest of the section we suppose given a chain $\sigma_{b}$ and $k \geqslant 1$ and focus on the computation of $d m m_{b}(k)$, that is, a bound on the number of deadlines that $\sigma_{b}$ can miss out of a $k$-sequence, i.e., $k$ consecutive activations. Similar to [10], we assume that there is at most one activation of an overload chain $\sigma_{a}$ in a $\sigma_{b}$-busy-window. As a result, we can without loss of generality consider our overload task chains as synchronous.

\section{A. Combinations for TWCA of task chains}

For the case where tasks are independent, a combination is defined as a set of overload tasks. The DMM computation based on this definition heavily relies on the fact that one overload activation impacts exactly one busy window. In the context of task chains, we have seen in the previous sections that one instance of a task chain $\sigma_{a}$ may span over several $\sigma_{b}$-busy-windows. As a result, the impact of one overload activation is not here limited to one $\sigma_{b}$-busy-window. We have however also shown that the execution an active segment of $\sigma_{a}$ is restricted to a single $\sigma_{b}$-busy-window. Hence our choice to define combinations based on active segments rather than tasks or task chains.

Definition 9. A combination $\bar{c}$ is a set of active segments w.r.t. $\sigma_{b}$ such that if two active segments of the same chain $\sigma_{a}$ are in $\bar{c}$ then they are part of the same segment of $\sigma_{a}$ w.r.t. $\sigma_{b}$.

Note that our definition excludes combinations which cannot execute within one $\sigma_{b}$-busy-window based on our definition of segment.

Example. There are four possible combinations of the active segments of chain $\sigma_{a}$ in Figure 1: $\left\{\left(\tau_{a}^{1}, \tau_{a}^{2}\right)\right\},\left\{\left(\tau_{a}^{3}\right)\right\},\left\{\left(\tau_{a}^{5}\right)\right\},\left\{\left(\tau_{a}^{1}, \tau_{a}^{2}\right),\left(\tau_{a}^{3}\right)\right\}$.

Definition 10. A combination $\bar{c}$ is schedulable (w.r.t. $\sigma_{b}$ ) if $\sigma_{b}$ is guaranteed not to miss any deadline in a $\sigma_{b}$-busy-window in which only the active segments in $\bar{c}$ execute (in addition to non-overload chains). Otherwise $\bar{c}$ is said to be unschedulable. 


\section{B. An ILP formulation for the DMM}

Having clarified the notion of combination that we use, we can now state our main theorem, similar to [10].

Theorem 3. Let us define $d m m_{b}(k)$ as

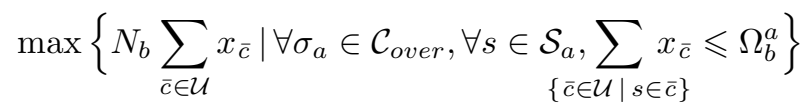

where

- $N_{b}$ is the maximum number of deadlines that $\sigma_{b}$ can miss in one busy window;

- $\mathcal{U}$ is the set of unschedulable combinations;

- $x_{\bar{c}}$ is the variable constraining the number of busy windows that could contain one activation of the $k$-sequence and suffer from an overload corresponding to $\bar{c} \in \mathcal{U}$;

- $\mathcal{S}_{a}$ denotes the set of active segments of $\sigma_{a}$;

- $\Omega_{b}^{a}$ is the maximum number of activations of $\sigma_{a}$ which could impact the considered $k$ activations of $\sigma_{a}$.

Then $d m m_{b}(k)$ is a DMM for $\sigma_{b}$.

The formal definition of $N_{b}$ and $\Omega_{b}^{a}$ is given below. Because $\mathcal{U}$ can be too large to be statically constructed, Section V-C discusses an efficient criterion to determine whether a combination is in $\mathcal{U}$. The $x_{\bar{c}}$ are the variables of our ILP problem.

Proof. Assume that we have $\Omega_{b}^{a}$ for all chains $\sigma_{a}$, i.e. the maximum number of activations of $\sigma_{a}$ which could impact the $k$-sequence. In the worst case, each active segment of $\sigma_{a}$ also impacts $\sigma_{b} \Omega_{b}^{a}$ times. As in Section III, we here also face a multi-dimensional knapsack problem where items correspond to unschedulable combinations and capacities to $\Omega_{b}^{a}$ for every line $s$ associated with an active segment of overload chain $\sigma_{a}$. So considering that $x_{\bar{c}}$ stands for the number of times that a combination $\bar{c}$ is used in the packing under consideration, we want to find the packing that maximizes the number of deadline misses of $\sigma_{b}$ - which is equal to the number of unschedulable combinations used multiplied by the maximum number of deadline misses due to each combination. This packing is constrained by the fact that active segments cannot be used in more combinations than is allowed by their corresponding $\Omega_{b}^{a}$.

Let us now formally define $N_{b}$ and $\Omega_{b}^{a}$.

\section{Lemma 3.}

$$
N_{b}=\#\left\{q \in\left[1, K_{b}\right] \mid B_{b}(q)-\delta_{b}^{-}(q)>D_{b}\right\}
$$

Proof. The proof proceeds exactly like that of Theorem 2 . $\square$

Lemma 4. The maximum number $\Omega_{b}^{a}$ of activations of $\sigma_{a}$ which could impact the considered $k$ activations of $\sigma_{a}$ is

$$
\Omega_{b}^{a}=\eta_{a}^{+}\left(\delta_{b}^{+}(k)+W C L_{b}\right)+1
$$

Proof. Clearly, activations of chain $\sigma_{a}$ which occur after the first instance of chain $\sigma_{b}$ in the $k$-sequence is activated and before the last activation in the $k$-sequence finishes may have an impact on the latencies in the $k$-sequence. There are at most $\eta_{a}^{+}\left(\delta_{b}^{+}(k)+W C L_{b}\right)$ such activations. In contrast, an instance of $\sigma_{a}$ which arrives after the last instance of chain $\sigma_{b}$ in the $k$ sequence has finished does not impact the $k$-sequence. Finally, we have assumed that there is at most one activation of $\sigma_{a}$ in a $\sigma_{b}$-busy-window so that at most one activation of $\sigma_{a}$ before the $k$-sequence can impact it.

\section{Criterion of schedulability}

As already mentioned, $\mathcal{U}$ can be too large to be statically constructed. We present here an efficient criterion to determine whether a combination $\bar{c}$ is in $\mathcal{U}$ or not. Let us reorganize Equation 1 for the multiple busy-time computation to show explicitly the contribution of the overload chains of a combination in the multiple busy time (and the latency) of $\sigma_{b}$.

$$
\begin{aligned}
B_{b}^{\bar{c}}(q)= & q \times C_{b} \\
& +\max \left(0, \eta_{b}^{+}\left(B_{b}^{\bar{c}}(q)\right)-q\right) \times C_{s_{b}^{\text {header }}} \text { if } \sigma_{b} \in \mathcal{A C} \\
& +\sum_{\sigma_{a} \in \mathcal{I} \mathcal{C}(b) \backslash \mathcal{C}_{\text {over }}} \eta_{a}^{+}\left(B_{b}^{\bar{c}}(q)\right) \times C_{a} \\
& +\sum_{\sigma_{a} \in \mathcal{A} \mathcal{A} \cap \mathcal{D C}(b)} \eta_{a}^{+}\left(B_{b}(q)\right) \times C_{s_{a, b}^{\text {header }}}+\sum_{s \in S_{a}^{b}} C_{s} \\
& +\sum_{\sigma_{a} \in \mathcal{S C} \cap \mathcal{D C}(b) \backslash \mathcal{C}_{\text {over }}} C_{s_{b}^{a}} \\
& +\sum_{\sigma_{a} \in \mathcal{C}_{\text {over }}} \sum_{s \in \mathcal{S}_{a}} C_{s} \times r_{s}^{\bar{c}}
\end{aligned}
$$

where $r_{s}^{\bar{c}}$ is a Boolean which holds exactly when $s \in \bar{c}$.

A combination $\bar{c}$ is schedulable if $B_{b}^{\bar{c}}(q)-\delta_{b}^{-}(q) \leqslant D_{b}$ for all $q \in\left[1, K_{b}\right]$. Now, let us define $L_{b}(q)$ as follows.

$$
\begin{aligned}
L_{b}(q) & =q \times C_{b} \\
& +\max \left(0, \eta_{b}^{+}\left(\delta_{b}^{-}(q)+D_{b}\right)-q\right) \times C_{s_{b}^{\text {header }}} \text { if } \sigma_{b} \in \mathcal{A C} \\
& +\sum_{\sigma_{a} \in \mathcal{I C}(b) \backslash \mathcal{C}_{\text {over }}} \eta_{a}^{+}\left(\delta_{b}^{-}(q)+D_{b}\right) \times C_{a} \\
& +\sum_{\sigma_{a} \in \mathcal{A} \mathcal{C} \cap \mathcal{D C}(b)} \eta_{a}^{+}\left(\delta_{b}^{-}(q)+D_{b}\right) \times C_{s_{a, b}^{\text {header }}}+\sum_{s \in S_{a}^{b}} C_{s} \\
& +\sum_{\sigma_{a} \in \mathcal{S C} \cap \mathcal{D C}(b) \backslash \mathcal{C}_{\text {over }}} C_{s_{b}^{a}}
\end{aligned}
$$

Then we now have a much simpler sufficient condition for schedulability: $\bar{c}$ is schedulable if

$$
\forall q \in\left[1, K_{b}\right], L_{b}(q)+\sum_{\sigma_{a} \in \mathcal{C}_{\text {over }}} \sum_{s \in \mathcal{S}_{a}} C_{s} \times r_{s}^{\bar{c}} \leqslant \delta_{b}^{-}(q)+D_{b}
$$

We have now shown how we can reuse the ILP solution of [10] for systems with task chains with limited changes.

\section{EXPERIMENTAL RESULTS}

We have experimented with a case study directly derived from industrial practice at Thales Research \& Technology. The system is a single-core processor scheduled according to SPP. Figure 4 shows the specified task set and the real-time attributes of each task. In the following experiments we focus on providing DMMs for $\sigma_{c}$ and $\sigma_{d}$. 


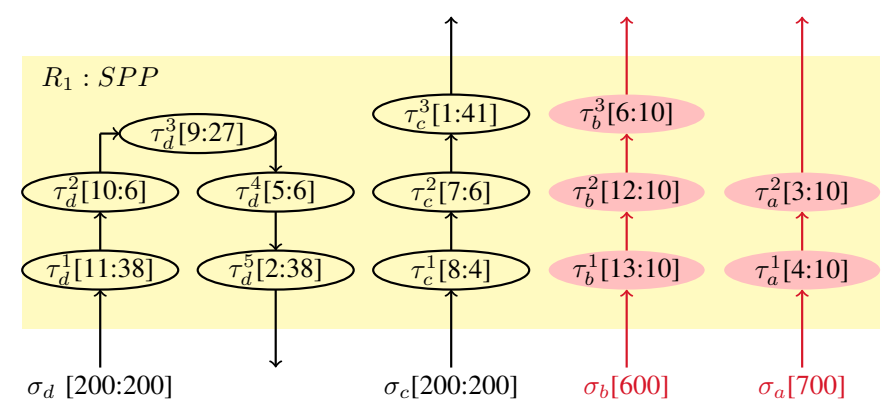

Figure 4. Model of our case study. We use the following notations: task chains are specified as $\sigma\left[\delta^{-}(2): D\right]$ and tasks with $\tau[\pi: C]$. Chains $\sigma_{c}$ and $\sigma_{d}$ are periodically activated while $\sigma_{a}$ and $\sigma_{b}$ are sporadic, overload chains.

Experiment 1. We first compute the worst-case latency $W C L$ of task chains $\sigma_{c}$ and $\sigma_{d}$ as described in Section IV. The analysis results show that the system is not schedulable as $\sigma_{c}$ can in the worst-case miss its deadline, see Table I.

\begin{tabular}{|c|c|c|}
\hline task chain & $W C L$ & $D$ \\
\hline$\sigma_{c}$ & 331 & 200 \\
\hline$\sigma_{d}$ & 175 & 200 \\
\hline
\end{tabular}

Table I

$W C L$ OF TASK CHAINS $\sigma_{c}$ AND $\sigma_{d}$

A second analysis, in which all overload chains are abstracted away, reveals that the system is schedulable and $\sigma_{c}$ meets its deadline if neither $\sigma_{a}$ nor $\sigma_{b}$ are activated. We thus perform TWCA as presented in this paper. The computed DMM of $\sigma_{c}$ is shown in Table II $-\sigma_{d}$ is schedulable and therefore does not need a DMM.

\begin{tabular}{|c|c|}
\hline task chain & DMM \\
\hline$\sigma_{c}$ & $d m m_{c}(3)=3, d m m_{c}(76)=4, d m m_{c}(250)=5$ \\
Table II \\
$d m m(k)$ FOR TASK CHAIN $\sigma_{c}$
\end{tabular}

Let us provide additional details resulting from this DMM computation. Both chains $\sigma_{a}$ and $\sigma_{b}$ arbitrarily interfere with $\sigma_{c}$ because neither has a task with a priority lower than 1 which is the lowest priority in $\sigma_{c}$. As a result $\sigma_{a}$ and $\sigma_{b}$ have only one segment, respectively $\left(\tau_{a}^{1}, \tau_{a}^{2}\right)$ and $\left(\tau_{b}^{1}, \tau_{b}^{2}, \tau_{b}^{2}\right)$. These two segments are also active segments because the priority of the tail task of chain $\sigma_{c}$ is lower than all priorities in these segments (see figure 4). Therefore no constraints on combining active segments are needed. Our set of combinations thus has three elements: $\bar{c}_{1}=\left\{\left(\tau_{a}^{1}, \tau_{a}^{2}\right)\right\}, \bar{c}_{2}=\left\{\left(\tau_{b}^{1}, \tau_{b}^{2}, \tau_{b}^{3}\right)\right\}$, and $\bar{c}_{3}=\left\{\left(\tau_{a}^{1}, \tau_{a}^{2}\right),\left(\tau_{b}^{1}, \tau_{b}^{2}, \tau_{b}^{3}\right)\right\}$. Based on the schedulability criterion we introduced in the previous section we conclude that $\bar{c}_{3}$ is the only unschedulable combination, so in this case the TWCA is fairly simple.

We now want to generalize the results obtained on our industrial case study, while preserving practical relevance. For that purpose, we arbitrarily modify the priority assignment so as to generate random systems with different scenarios.

Experiment 2. We arbitrarily assign priorities to show the impact of priority assignments on the schedulability and the deadline miss models. In this experiment we randomly choose 1000 assignments to test our analysis intensively. Figure 5 shows $d m m_{c}(10)$ and $d m m_{d}(10)$. Notice first that out of
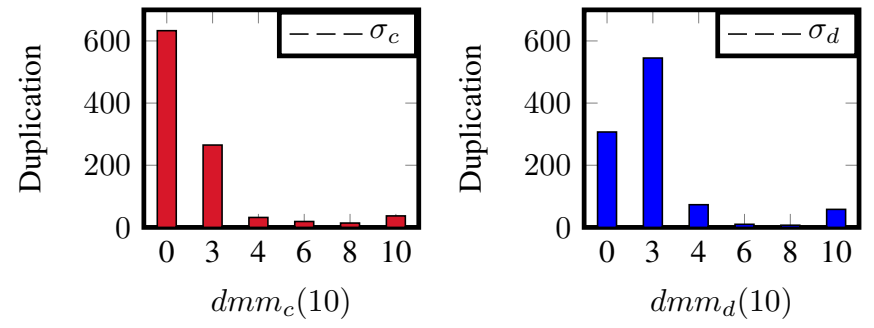

Figure 5. $d m m_{c}(10)$ and $d m m_{d}(10)$

the 1000 assignments generated, chain $\sigma_{c}$ is schedulable (misses no deadline) 633 times. More interestingly, chain $\sigma_{d}$ is schedulable only 307 times out of 1000. TWCA in that case is very useful as for more than 500 of the remaining systems it can guarantee that no more than 3 out 10 deadlines can be missed. Note that we have repeated our experiment 30 times and observed similar results.

\section{CONCLUSION}

In this paper we present the first method for computing end-to-end deadline miss models for systems with task dependencies, using Typical Worst-Case Analysis (TWCA). This bounds the number of potential deadline misses in a given sequence of activations of a task chain. Our approach addresses uniprocessor systems with Static Priority Preemptive scheduling. We show how state-of-the-art TWCA can be extended using recent results in the analysis of hard realtime systems with task dependencies. Specifically, we show how we can formulate our problem as a knapsack problem. Our approach is validated on a realistic case study inspired by industrial practice and synthetic variants of it.

This paper is an important step towards using TWCA for the practical design of distributed embedded systems.

\section{REFERENCES}

[1] G. Bernat, A. Burns, and A. Llamosí. Weakly hard real-time systems. IEEE Trans. Computers, 50(4):308-321, 2001.

[2] J. C. P. Gutiérrez and M. G. Harbour. Schedulability analysis for tasks with static and dynamic offsets. In Proceedings of RTSS'19, pages 2637, 1998.

[3] J. C. P. Gutiérrez and M. G. Harbour. Exploiting precedence relations in the schedulability analysis of distributed real-time systems. In Proceedings of RTSS'99, pages 328-339, 1999.

[4] M. Hamdaoui and P. Ramanathan. A dynamic priority assignement technique for streams with (m, k)-firm deadlines. IEEE Trans. Computers, 44(12):1443-1451, 1995.

[5] Z. A. H. Hammadeh, S. Quinton, and R. Ernst. Extending typical worst-case analysis using response-time dependencies to bound deadline misses. In Proceedings of EMSOFT'14, pages 10:1-10:10, 2014.

[6] P. Kumar and L. Thiele. Quantifying the effect of rare timing events with settling-time and overshoot. In Proceedings of RTSS'33, pages 149-160, 2012.

[7] M. Moy and K. Altisen. Arrival curves for real-time calculus: The causality problem and its solutions. In Proceedings of TACAS'10, pages 358-372, 2010.

[8] S. Quinton, M. Hanke, and R. Ernst. Formal analysis of sporadic overload in real-time systems. In Proceedings of DATE'12, pages 515520. IEEE, 2012.

[9] J. Schlatow and R. Ernst. Response-time analysis for task chains in communicating threads. In Proceedings of RTAS'16, 2016.

[10] W. Xu, Z. A. H. Hammadeh, A. Kröller, S. Quinton, and R. Ernst. Improved deadline miss models for real-time systems using typical worst-case analysis. In Proceedings of ECRTS'15, pages 247-256, 2015. 